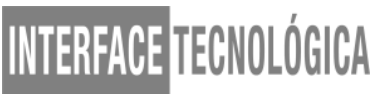

\title{
REVISÃO SOBRE O USO DE TÉCNICAS DE ENCONTRABILIDADE NA WEB
}

\section{REVIEW ON THE USE OF TECHNIQUES OF FINDABILITY ON WEB}

\author{
Rafael Gabriel Marsico Gonçalves - rg.marsico@ gmail.com \\ Erick Eduardo Petrucelli - erickpetru@gmail.com \\ Faculdade de Tecnologia de Taquaritinga (FATEC) - SP - Brasil
}

\begin{abstract}
RESUMO
Os recentes avanços tecnológicos possibilitaram o acesso à informação, recuperação de dados, assim como o uso e apropriação da informação e relação entre dados de diferentes dispositivos e sistemas. Diante deste cenário, surgiu o termo Findability, que mostra a facilidade em se encontrar qualquer informação a respeito de algo ou alguém a partir de qualquer local e a qualquer momento, usando ferramentas de busca. Este trabalho teve como objetivo desenvolver uma pesquisa literária de técnicas em Encontrabilidade e suas aplicações, assim como verificar sua utilização. O objetivo da pesquisa foi atingido, pois foi possível fazer um levantamento bibliográfico, entre publicações de diversos autores da área, e verificar que o objeto da pesquisa já vem fazendo parte da vida dos usuários, pois a Encontrabilidade no ambiente informacional, leva os usuários da Web a conseguirem encontrar as informações que buscam, a qualquer momento, de qualquer lugar, desde que estejam conectados a Internet.
\end{abstract}

Palavras-chave: Findability, Tecnologia da Informação.

\begin{abstract}
Recent technological advances, the possibility of access to information, data recovery, such as the use and adequacy of information and the relationship between data from different devices and devices. In the face of this scenario, one searches for what is a problem, and shows a facility in any information about something or someone from any place and at any time, using search tools. This work had as objective to develop a literary research of techniques in Findability and and its applications, as well as verify their use. The objective of the research was reached, because it was possible to make a bibliographical survey, among publications of several authors of the area, and to verify that the research object is already part of the life of the users, because the Findability in the informational environment, Web to be able to find the information they seek, at any time, from anywhere, as long as they are connected to the Internet
\end{abstract}

Keywords: Findability. Information Technology 


\section{INTRODUÇÃO}

A Web (World Wide Web) é um meio de fácil acesso à informação, entretanto nem sempre os usuários sabem exatamente qual URL (Uniform Resource Locator) deverão utilizar para serem direcionados à página que contenha o conteúdo exato de seus interesses. Diante disso, as pessoas recorrem às ferramentas de busca para encontrar páginas relacionadas ao assunto no qual elas estão interessadas (DAL'EVEDOVE; FIGUEIRA, 2017).

Nas últimas décadas, notou-se o aparecimento de diversas nomenclaturas na literatura para a Web, que foram influenciadas por tecnologias e/ou práticas específicas, como, por exemplo: Web 2.0, Web Social, Web 3.0, Web Semântica, Web Social Semântica, Web Pragmática, Web NG (Web da Nova Geração), Web Profunda, entre outras (VECHIATO, 2013). De acordo com Vechiato e Vidotti (2014a), recentemente, também, avanços tecnológicos possibilitaram o acesso à informação, recuperação de dados, assim como o uso e apropriação da informação e relação entre dados de diferentes dispositivos e sistemas, sendo esse o novo cenário para a tecnologia da informação. Diante disso, surgiu o termo Findability, que demonstra a facilidade em se encontrar qualquer informação a respeito de algo ou alguém a partir de qualquer local e a qualquer momento (MARCOS, 2007).

Segundo Fernandes et al. (2016), a Internet vem mudando o cotidiano das pessoas, sendo que mais de $50 \%$ das casas brasileiras possuem acesso à Internet. Sua utilização está destinada a diversas finalidades, como acesso a informação, diversão, meios de relacionamento, compras, entre outros. Os dados, cujas tendências são de crescimento, nos revelam a importância que os web sites trazem para a vida da população brasileira, e este ambiente deve facilitar a Encontrabilidade da informação disponível.

O termo Encontrabilidade, traduzido do inglês Findability, foi definido preliminarmente por Peter Morville (2005), considerando seu diálogo com os estudos relacionados à recuperação da informação na Ciência da Informação e sua relevância para os ambientes informacionais busca-se sustentar um conceito funcional para Encontrabilidade a fim de ser incorporado no campo científico da tecnologia da informação.

Diante disso, busca-se compreender o desenvolvimento da Web e quais as perspectivas para promover a Encontrabilidade da informação, tendo como respaldo o conceito de mediação infocomunicacional nesse contexto e enfatizando os processos informacionais e suas características (VECHIATO; VIDOTTI, 2014a). 


\section{WTERERAGETECWNOLOGGCA}

Este trabalho teve como objetivo desenvolver uma pesquisa literária de técnicas em Encontrabilidade e suas aplicações, assim como verificar sua utilização.

\section{TECNOLOGIA DA INFORMAÇÃO E ENCONTRABILIDADE}

O desenvolvimento científico e tecnológico, desde o final da Segunda Guerra Mundial até os dias atuais, impulsionou mudanças na sociedade, principalmente no que diz respeito ao acesso à informação e à construção do conhecimento (VECHIATO; VIDOTTI, 2014).

O surgimento da World Wide Web (WWW e Web), em conjunto com a evolução das Tecnologias de Informação e Comunicação (TIC) e uso da Internet mudou a sociedade, que já pode ser considerada a "Sociedade da Informação". Diante desses fatos, a geração de dados aumentou de tal forma que trouxe grandes preocupações em relação à organização, a representação, recuperação, Encontrabilidade e apropriação da informação pelo usuário. A evolução da Web fez com que a participação dos usuários na produção de conteúdo aumentasse efetivamente, ou seja, o usuário passou a ser produtor e não apenas um consumidor de informações nos espaços digitais (SANCHEZ, 2016). Nesse contexto, para Schiessl (2007), a criação e a distribuição da informação podem ocorrer em qualquer lugar do mundo, a qualquer hora, por qualquer pessoa, desde que ela esteja conectada à Internet. Desta forma a Internet pode ser considerada uma biblioteca digital, onde uma pessoa pode divulgar livremente qualquer informação, verdadeira ou não, sem que exista controle algum sobre seu conteúdo, sem sequer a estruturação e organização necessárias para sua recuperação (FARIAS 2011).

Sabe-se que realizar as tarefas de pesquisa exploratória, ainda é um desafio, pois em geral, o conhecimento gerado não é estruturado e há problemas de falta de consistência. $\mathrm{Na}$ última década, alguns trabalhos objetivaram promover uma abordagem semântica às iniciativas da Web 2.0 através da criação de ontologias colaborativas (NOY, 2008). Estudos sugerem que, quanto mais o usuário se familiarizar à organização de um site, melhor é a qualidade da Encontrabilidade (WANG; FILGUEIRAS, 2008).

Acredita-se que uma organização que leve em conta o contexto pode ser favorável a Encontrabilidade no caso de uma pesquisa exploratória. Nesse sentido, o CSCOS (Common Sense Context Organization Scheme), produz uma organização que leva em conta o contexto do assunto tratado pelos textos do site, contexto esse detectado com apoio de uma Base de Conhecimento de Senso Comum (WANG; FILGUEIRAS, 2010). Ainda segundo os autores, as características comuns dos itens de conteúdo definem o esquema de organização, influenciando 


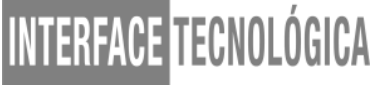

o agrupamento lógico desses itens, onde uma organização eficaz auxilia o usuário a encontrar o que procura.

Diante desse contexto, a linguagem utilizada é de suma importância, pois, qualquer que seja a linguagem utilizada é necessário que seja estabelecida uma relação entre a linguagem natural usada pelo usuário e a linguagem que é usada pelo sistema de recuperação da informação. Para tanto, são estruturados instrumentos de controle de termos, buscando estabelecer um vocabulário comum, que visa facilitar o processo de comunicação entre o usuário e o sistema (FARIAS 2011).

Nesse contexto, Dal'Evedove e Figueira, (2017), relataram a existência de algumas etapas específicas de consumo de informações até chegar à pesquisa feita pelo usuário, sendo a coleta a primeira etapa, seguida por armazenamento, extração, indexação, classificação semântica e por link, finalizando com a pesquisa. De acordo com Farias (2011), nas últimas décadas, diversos sistemas de organização e recuperação de informação vêm sendo indicados e utilizados para classificar a informação, independente do formato da mídia, dentre eles podemos destacar uma simples lista de cabeçalho de assuntos, como as tradicionais ferramentas de indexação e recuperação, assim como os instrumentos para contextos e aplicações mais específicas, como as taxonomias e as ontologias (MORAES; OLIVEIRA, 2010).

Ao considerar os processos informacionais desenvolvidos no cenário evolutivo da Web, entende-se que as formas de produção, representação e organização, estão aliadas às possibilidades de recuperação, acesso, uso e apropriação, onde se estabelece uma forte relação entre alguns elementos que constituem a interação, o compartilhamento e a colaboração, como por exemplo, ambientes, sistemas de informação e sujeitos informacionais (VECHIATO; VIDOTTI, 2014b). De acordo com estes autores, a Encontrabilidade surge desta perspectiva, como um importante elemento que se situa entre a efetividade do funcionamento dos ambientes informacionais e as características do sujeito.

O conceito de Encontrabilidade utilizado neste estudo resulta do cenário paradigmático que privilegia o acesso à informação com ênfase nas necessidades, competências, comportamentos e características dos usuários para a organização da informação. (VECHIATO; VIDOTTI, 2014a, 2014b). 


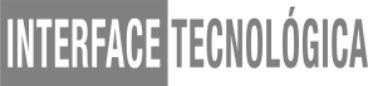

Para Vechiato e Vidotti (2009), uma das formas de se promover a Encontrabilidade em um ambiente de informação, é projetá-lo a partir da arquitetura da informação, a qual agrega estudos como usabilidade, acessibilidade e comportamento informacional. Esses estudos aplicados em conjunto, fornecem subsídios tanto práticos quanto teóricos para a construção de ambientes informacionais digitais (VECHIATO; VIDOTTI, 2014a).

\section{PROCEDIMENTOS METODOLÓGICOS}

Este trabalho foi um estudo de revisão de literatura, baseado em pesquisa utilizando os termos "Encontrabilidade" e "Findability" nos bancos de dados encontrados na ferramenta de busca Google Acadêmico, e revisão não sistemática de artigos de revistas e periódicos e monografias.

\section{RESULTADOS E DISCUSSÃO}

De acordo com Farias, (2011), o termo Encontrabilidade refere-se à qualidade de ser localizável uma informação. Em relação a este item, pode-se avaliar até que ponto um determinado objeto é encontrado ou localizado facilmente, enquanto que em relação a sistema, pode-se avaliar o quanto um ambiente físico ou digital suporta a navegação e a recuperação de dados (MARTIN, 2004). Nesse sentido, para Morville (2005), pode-se encontrar alguém ou alguma coisa de qualquer lugar a qualquer momento, usando tecnologias como GPS (Sistema de posicionamento Global) e RFID (Identificação por Radio frequência), celular e outras tecnologias de localização, para o autor, a Encontrabilidade está associada à arquitetura da informação, usabilidade e otimização de sites da $W e b$, de acordo com a figura a seguir:

Figura 1: Relação da Encontrabilidade e outras disciplinas da Web 


\section{WNTEREHEG TEENOLOGGCA}

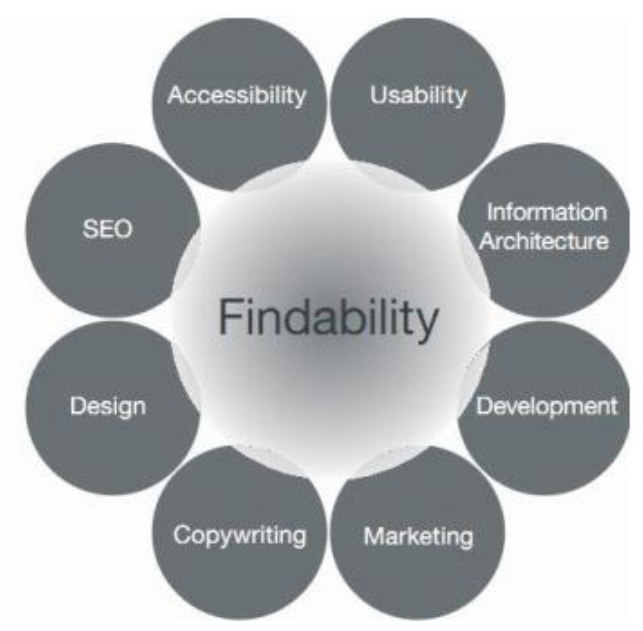

Fonte: Walter, (2009); Farias, (2011).

\begin{tabular}{|l|l|}
\hline Findability & Encontrabilidade \\
\hline SEO & $\begin{array}{l}\text { Search Engine } \\
\text { Optimization }\end{array}$ \\
\hline Accessibility & Acessibilidade \\
\hline Usability & Usabilidade \\
\hline $\begin{array}{l}\text { Information } \\
\text { Architecture }\end{array}$ & $\begin{array}{l}\text { Arquitetura da } \\
\text { Informação }\end{array}$ \\
\hline Development & Desenvolvimento \\
\hline Marketing & Marketing \\
\hline Design & Design \\
\hline
\end{tabular}

De acordo com Farias (2011), a Encontrabilidade se relaciona com outras disciplinas e áreas, tais como Marketing, Design, Acessibilidade, Redação Publicitária, SEO (Search Engine Optimization) e desenvolvimento de sites. Segundo o autor, a Encontrabilidade já fazia parte do universo da Web mesmo sem ser apresentada aos seus usuários, entretanto com o aumento do número de informações na $W e b$, fez-se necessária a exatidão nos processos de busca.

De acordo com os autores Caro, Blass e Spagnolo (2012), fornecer uma funcionalidade de busca padrão Web seria insuficiente quando se tem uma grande quantidade de dados de conteúdo a serem indexados e consultados, pois a função de pesquisa requer que o usuário ou sujeito informacional solicite exatamente as mesmas palavras-chave utilizadas originalmente para definir o conteúdo de seus artigos. Se a combinação não for precisa, a informação tende a permanecer, obviamente, não localizada, sendo necessária uma forma de adicionar resultados semelhantes e contextualmente relevantes que podem gerar o interesse dos usuários. O Modelo de Encontrabilidade da Informação (MEI) proposto por Vechiato e Vidotti (2014a) pode ser aplicado em quaisquer sistemas, ambientes ou arquiteturas da informação, podendo ser identificado em diversos trabalhos. Para esses autores, a Encontrabilidade da Informação "[...] sustenta-se fundamentalmente na interseção entre as funcionalidades de um ambiente informacional e as características dos sujeitos informacionais" (Vechiato e Vidotti (2014a, p. 164)). Dentro do contexto, para Custódio e Vechiato (2013) o conceito de 'Encontrabilidade da Informação' se alia ao de 'Mediação Infocomunicacional' e consideram que os profissionais da informação, os profissionais de informática e os próprios usuários de ambientes informacionais são mediadores, pois todos eles exercem um papel fundamental nesses ambientes, influenciando a Encontrabilidade da Informação de forma substancial. 


\section{WNTEREHESTEENOLOGGCA}

\section{CONCLUSÃO}

Conclui-se que a Encontrabilidade é uma ferramenta importante para se encontrar algum dado especifico e/ou determinado que vá gerar um conjunto de Informações, pois traz para o usuário o acesso facilitado, e rápido à aquilo que se busca na internet. O objetivo da pesquisa foi atingido, pois foi possível fazer um levantamento bibliográfico, entre publicações de diversos autores da área, e verificar que o objeto da pesquisa já vem fazendo parte da vida dos usuários, pois a Encontrabilidade no Ambiente Informacional, leva os usuários da Web a conseguirem encontrar as informações que buscam, a qualquer momento, de qualquer lugar, desde que estejam conectados à Internet.

\section{CONSIDERAÇÕES FINAIS}

As Tecnologias estão sempre ligadas a Ciência da Informação e em seu desenvolvimento, envolvendo produção, organização, armazenamento e recuperação de dados nos mais variados ambientes informacionais. Neste contexto, faz-se necessário o desenvolvimento de estudos que visem melhorar o acesso à informação para auxiliar na construção do conhecimento (VECHIATO; VIDOTTI, 2014), tornando-se relevante o estudo de teorias, métodos, instrumentos e metodologias que possam contribuir para futuros projetos e para uma melhor avaliação de ambientes informacionais, de modo a facilitar a Encontrabilidade da Informação disponível pelos sujeitos com base em suas necessidades Informacionais e suas intencionalidades, amenizando as dificuldades nessa área (VECHIATO; OLIVEIRA; VIDOTTI, 2016).

\section{REFERÊNCIAS}

CARO, S.; BLASS, N. D.; SPAGNOLO, L. Arquitetura de informação: Como melhorar a Encontrabilidade do conteúdo do seu site. Museums and the Web, 2012. Disponível em:<http://br.masternewmedia.org/2010/09/01/arquitetura_de_informacao_como_melhorar_a _encontrabilidade.htm>Acesso em: 4 de Junho de 2017.

CUSTÓDIO, N. C.; VECHIATO, F. L. Mediação Infocomunicacional no contexto da Encontrabilidade da informação: uma análise do processo de auto arquivamento no repositório institucional da Universidade Federal do Rio Grande do Norte. Biblionline, João Pessoa, v. 12, n. 1, p. 3- 13, 2016. Disponível em:< file:///D:/Downloads/28235-62955-1-PB.pdf> Acesso em: 26 de Maio de 2017.

DAL'EVEDOVE, B. A. O.; FIGUEIRA, L. B. Encontrabilidade digital e web 3.0: uma web estruturada para comunicação entre máquinas e seres humanos. Interface Tecnológica. V. 14,
n.
1,
p.
42
52.
2017.
Disponível
em: 


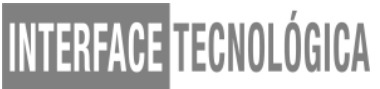

<http://159.203.166.88/index.php/interfacetecnologica/article/view/148>. Acesso em: 28 ago. 2017.

FARIAS R. A. N. Análise da Encontrabilidade do website do departamento de biblioteconomia da UFRN. Monografia (Graduação em Biblioteconomia). Universidade Federal do Rio Grande do Norte. 2011.

FERNANDES, W. M., SILVANA APARECIDA BORSETTI GREGORIO VIDOTTI, S. A. B. G.; VECHIATO, F. L. arquitetura e Encontrabilidade da informação: análise de um ambiente de e-commerce com a tecnologia de eye tracking. 2016. Disponível em:<http://gpnti.marilia.unesp.br:8085/index.php/3DTI/3dti/paper/view/393> Acesso em 26 de Março de 2017.

MARCOS, M. Information Architecture \& Findability: Peter Morville interview. El profesional de la información, v. 16, n. 3, p. 268-269, maio/jun. 2007. Disponível em: < http://www.elprofesionaldelainformacion.com/contenidos/2007/mayo/12.pdf> Avesso em: 25 de Março de 2017.

MORAES, A. F.; OLIVEIRA, T. M. Experiências relacionadas ao levantamento de teses e dissertações. Informação \& Sociedade: Estudos, v. 20, n. 1, p. 73 - 81.2010

MORVILLE, P. Ambient Findability. Sebastopol: O’Really, 2005.

MORVILLE, P. Ambient Findability: Findabilitys hacks. A List Apart Magazine. 2005. Disponível em : <https://alistapart.com/article/ambientfindability> Acesso em: 30 de maio de 2017.

NOY, N. F.; CHUGH, A.; ALANI, H. The CKC Challenge: Exploring tools for collaborative knowledge construction. IEEE Intelligent Systems, vol. 23, no. 1, pp. 64-68, Jan/Feb., 2008.

SANCHEZ. CONTRIBUIÇÕES DAS TECNOLOGIAS SEMÂNTICAS PARA A ENCONTRABILIDADE DA INFORMAÇÃO: um estudo em ambientes informacionais digitais colaborativos - Wikis. DTI - III Encontro Internacional de Dados, Tecnologia e Informação.Marília-SP.2016.Disponível

em:<http://gpnti.marilia.unesp.br:8085/index.php/3DTI/3dti/paper/viewFile/392/164>Acesso em: 2 de Maio de 2017.

SCHIESSL, M. Ontologia: o termo e a ideia. R. Eletr. Bibliotecon. Ci. Inf., Florianópolis, n. 24 , p. $172-181,2^{\circ}$ sem. 2007.

VECHIATO, F. L. Encontrabilidade da informação: contributo para uma conceituação no campo da ciência da informação. 2013. 206 f. Tese (Doutorado em Ciência da Informação) Faculdade de Filosofia e Ciências, Universidade Estadual Paulista, Marília, 2013. Disponível em: $<$ https://www.marilia.unesp.br/Home/PosGraduacao/CienciadaInformacao/Dissertacoes/T ese_de_Doutorado_-_Fernando_Luiz_Vechiato.pdf>. Acesso em: 25 de Março de 2017.

VECHIATO, F. L.; OLIVEIRA, H. P. C.; VIDOTTI, S. A. B. G. Arquitetura da Informação Pervasiva e Encontrabilidade da informação: instrumento para a avaliação de ambientes informacionais híbridos. XVII Encontro Nacional de Pesquisa em Ciência da Informação (XVII ENANCIB). $\quad$ Bahia, 2016. Disponível em: 


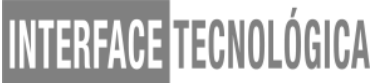

http://200.20.0.78/repositorios/bitstream/handle/123456789/3611/2016_GT8-

CO_27.pdf?Sequence=1>. Acesso em 26 de Agosto de 2017.

VECHIATO, F. L.; VIDOTTI, S. A. B. G. Encontrabilidade da Informação. 1. ed. São Paulo: Cultura Acadêmica, 2014a. (Coleção PROPG Digital- UNESP). ISBN 9788579835865. Disponível em: <http://hdl.handle.net/11449/126218>. Acesso em 23 de Junho de 2017.

VECHIATO, F. L.; VIDOTTI, S. A. B. G. Encontrabilidade da Informação: Atributos e Recomendações para Ambientes Informacionais Digitais. Informação \& Tecnologia (ITEC): Marília/João Pessoa, 1(2): 42-58, jul./dec., 2014b.

WALTER, A. Construindo websites que todos encontram. Rio de janeiro - RJ. Alta books. 2009.

WANG, W. S.; FILGUEIRAS, L. CSCOS. Uso de uma base de conhecimento de Senso Comum em projetos de Arquitetura da Informação de web sites. IHC 2008 - VII Simpósio sobre Fatores Humanos em Sistemas Computacionais. 2008.

WANG, W. S.; FILGUEIRAS, L. CSCOS - Organização de Conteúdo Orientada por Senso Comum. IHC 2010 - IX Simpósio sobre Fatores Humanos em Sistemas Computacionais. 2010. 